\title{
Got nerve? Autonomic innervation of the human liver
}

\section{Romil Saxena $^{1}$}

Received: 13 March 2020 / Revised: 13 March 2020 / Accepted: 17 March 2020 / Published online: 11 May 2020

(C) Springer-Verlag GmbH Germany, part of Springer Nature 2020

Literature on innervation of the human liver is not prolific. Although much may be extrapolated from animal studies, it is well acknowledged that there are significant differences in hepatic innervation between species [1]. The lack of substantial studies is however not surprising, given the difficulty of visualizing nerve fibers in routine pathologic sections of the liver, and comparable difficulty in designing studies that may reliably elucidate the anatomical, functional, and spatial properties of innervation in the human liver.

In contrast to literature on the subject, innervation of the liver itself is prolific, comprising all elements of the autonomic nervous system, including cholinergic, aminergic, peptidergic, and nitrergic fibers. That the liver is a veritable "bag of nerves" is not surprising $[1,2]$, given the role the autonomic nervous system plays in all aspects of mammalian function, of which the liver performs all, and then some.

Postganglionic sympathetic fibers are derived from splanchnic nerves originating in the celiac and the superior mesenteric ganglia and are more abundant around the hepatic artery. Postganglionic parasympathetic fibers are derived from ganglia located in the hepatic hilum and portal tracts. Neuropeptides, representing the peptidergic component, co-localize with neurotransmitters in both aminergic and cholinergic nerves. These neuropeptides include neuropeptide Y (NPY), substance P (SP), vasoactive intestinal peptide (VIP), calcitonin gene-related peptide (CGRP), glucagon-like peptide (GLP), somatostatin $(\mathrm{SOM})$, neurotensin $(\mathrm{NT})$, galanin (GAL), and serotonin. The nitrergic component is represented by intrahepatic nerves containing nitric oxide (NO), or neuronal nitric oxidase synthase (nNOS), the enzyme that catalyzes the formation of nitric oxide.

Romil Saxena

rsaxena@iupui.edu

1 Department of Pathology and Laboratory Medicine, Indiana University School of Medicine, 350 West 11th Street, Room 4014, Indianapolis, IN 46202, USA
Autonomic nerves enter the liver through the hilum, forming an anterior plexus around the hepatic artery, and a posterior plexus around the portal vein and bile duct. This rich portal innervation maintains flow of blood and bile, directly by maintaining tone of the vascular and biliary systems and indirectly by regulating sinusoidal flow, which is an essential determinant of optimal metabolic function of the liver [3, 4]. Whereas innervation of the portal tracts is uniform across species, lobular innervation is variable. In man, aminergic and peptidergic fibers have been shown to course through the lobule along the space of Disse, coming in close contact with hepatocytes and stellate cells, which suggests direct autonomic control of sinusoidal blood flow and hepatocellular metabolism. Similar lobular innervation has not been demonstrated in mice and rat livers; metabolic control is postulated to be achieved by either diffusion of neurotransmitters, regulation of sinusoidal blood flow by modulating tone of portal vessels, and/or transmission of signals through gap junctions between hepatocytes [1].

The autonomic nervous system plays a substantiative role in cholangiocyte pathophysiology. There is experimental evidence to suggest that it modulates output of bile salts, cholesterol, and lipid in response to somatostatin and feeding, as well as cell death and proliferation of cholangiocytes in response to cholestasis or liver injury. In this context, the article by Zanchi et al. in this issue of Virchows Archiv is pertinent, as for the first time, it demonstrates the spatial relationships between nerve fibers and the very proximal components of the biliary tree, namely, the canals of Hering, bile ductules, and interlobular bile ducts.

Bile secreted by hepatocytes into bile canaliculi makes its way through the lobule toward the portal tract, draining into the interlobular bile ducts via the canals of Hering. First described and uncannily illustrated by Ewald Hering in 1867 [5], these eponymous canals are the smallest and the most proximal portion of the biliary tree with a cholangiocyte lining, albeit incomplete. The canals of Hering are trough-like structures lined partially by cholangiocytes and partially by hepatocytes [6], thus representing the de facto interface of 
hepatocytes and cholangiocytes. It also makes biological sense that they form the niche that harbors bipotential hepatic progenitor cells, which contingent to the need of the hour and presence of an appropriate proliferative milieu, have the capacity to differentiate either into hepatocytes or cholangiocytes.

The autonomic nervous system has been shown to play a vital role in the proliferation of oval cells in the rat liver, and in stem cell regulation of hematopoiesis in the bone marrow. Might the autonomic nervous system similarly regulate regeneration in the human liver through its interaction with progenitor cells in the canals of Hering? Ductular reactions arise at the edges of portal tracts, and in the vicinity of canals of Hering in almost all chronic liver diseases, and appear to represent a regenerative attempt of the liver to compensate for loss of hepatocytes or cholangiocytes. Unfortunately, ductular reactions are associated with development of progressive fibrosis and scarring $[7,8]$. If ductular reactions are indeed orchestrated by the autonomic nervous system, can the signal be abrogated to prevent subsequent fibrosis and scarring? Might the processes of liver regeneration be maneuvered in a direction that is beneficial to human health, by coaxing the hepatocellular pathway of regeneration over the cholangiocytic, in chronic liver diseases as well as in acute liver failure due to submassive necrosis? And if so, might it be done by exclusively targeting the autonomic nervous system of the liver, without engaging the systemic or splanchnic systems with their unintended consequences? Is there a hepatic equivalent of the enteric autonomic system, independent from central control?

"If you answer one question, it will lead you to another, and another, and another. It's like peeling an onion." - with apologies to Lemony Snicket [9].

Author contributions Romil Saxena is responsible for literature search and drafting the manuscript.
Data availability Not applicable.

\section{Compliance with ethical standards}

Conflict of interest The author declares that he has no conflict of interest.

Ethics approval Not applicable.

Consent to participate/publish Not applicable.

\section{References}

1. McCuskey RS (2004) Anatomy of efferent hepatic nerves. Anat Rec A Discov Mol Cell Evol Biol 280:821-826

2. Bioulac-Sage P, Lafon ME, Saric J, Balabaud C (1990) Nerves and perisinusoidal cells in human liver. J Hepatol 10:105-112

3. Lautt WW (1983) Relationship between hepatic blood flow and overall metabolism: the hepatic arterial buffer response. Fed Proc 42:1662-1166

4. Lautt WW (1977) The hepatic artery: subservient to hepatic metabolism or guardian of normal hepatic clearance rates of humoral substances. Gen Pharmacol 8:73-78

5. Hering E (1867) Ueber den Bau der Wirbelthierleber. Archiv fur mikroskopische Anatomie 37:88-118

6. Saxena R, Theise ND, Crawford JM (1999) Microanatomy of the human liver - exploring the hidden interfaces. Hepatology 30:1339-1346

7. Williams MJ, Clouston AD, Forbes SJ (2014) Links between hepatic fibrosis, ductular reaction, and progenitor cell expansion. Gastroenterology. 146:349-356

8. Sato K, Marzioni M, Meng F, Francis H, Glaser S, Alpini G (2019) Ductular reaction in liver diseases: pathological mechanisms and translational significances. Hepatology. 69:420-430

9. Snicket L (2006) The end. HarperCollins, New York

The submitted work is original, has not been previously published in part or full, and has not been submitted to another journal for consideration.

Publisher's note Springer Nature remains neutral with regard to jurisdictional claims in published maps and institutional affiliations. 